\title{
Automatic Classification of Linear Structures in Mammographic Images
}

\author{
Reyer Zwiggelaar ${ }^{1}$, Christopher J. Taylor ${ }^{2}$, and Caroline R. M. Boggis ${ }^{3}$ \\ 1 Division of Computer Science \\ University of Portsmouth, Portsmouth, UK \\ reyer.zwiggelaar@port.ac.uk \\ 2 Wolfson Image Analysis Unit \\ University of Manchester, Manchester, UK \\ 3 Greater Manchester Breast Screening Service \\ Withington Hospital, Manchester, UK
}

\begin{abstract}
Certain kinds of abnormalities in x-ray mammograms are associated with specific anatomical structures - in particular, linear structures. This association can, in principle, be exploited to improve the specificity and sensitivity with which the abnormalities can be detected. We compare annotated and the automatic detection of the scale and orientation associated with linear structure in mammograms. We investigate methods of classifying the detected structures into anatomical classes (spicules, vessel, duct, fibrous tissue etc) from their crosssectional profiles. Automatic (linear and non-linear) classification results are compared with expert annotations using receiver operating characteristic analysis. We show that useful discrimination between anatomical classes is achieved. Some of this relies on simple attributes such as the width and contrast of the profile, but there is also important information carried by the shape of the profile.
\end{abstract}

\section{Introduction}

The UK Breast Screening Programme alone generates 1.5 million mammograms per annum. Potential malignancies can be detected from subtle abnormalities in radiographic appearance but it is known that radiologists fail to detect a significant proportion of these abnormalities. It has been shown that their performance would improve if they were prompted with the possible locations of abnormalities $[1,2,3,4,5]$. The abnormalities of interest include microcalcification clusters, masses, spiculated lesions, asymmetry and architectual distortions [6]. Normal mammograms also contain a variety of linear structures: vessels, ducts, fibrous tissue, skinfolds, edges and others that are difficult to classify anatomically. In abnormal mammograms linear structures called 'spicules' may also be present. Abnormalities are non-accidentally associated with these linear structures. For example, microcalcifications are more likely to imply malignancy if they are located in ducts [7] and spicules are always associated with lesions (called spiculated lesions). Each type of linear structure has a characteristic appearance which we hypothesize should be reflected in the cross-sectional intensity profiles. 
Spiculated lesions can be detected from the characteristic arrangement of linear structures (the spicules) in a radial pattern $[1,4,5]$. This is, however, less than perfect because other linear structures can be accidentally arranged in similar patterns in normal mammograms. We suggest that these methods could be made more specific by applying them to just those linear structures that have a high probability of being spicules. Linear structures can be detected using various approaches [8]. Here we investigate the ability to classify detected structures into anatomical classes using their cross-sectional intensity profiles, with particular emphasis on detecting spicules. Anatomical classification has been developed and tested using real screening mammograms, annotated by an expert radiologist. Raw intensity profiles are extracted and normalised for intensity and scale. The dimensionality of the observations is reduced using principal component analysis (PCA) and both linear and non-linear classification are investigated.

\section{Mammographic Data}

We will be giving a comparison between the width and orientation as obtained from the Line Operator approach [8] and those same parameters as provided by a radiologist based on real mammographic data (in [8] the comparison was based on synthetic data).

\subsection{Anatomical Annotations}

The Line Operator was applied to 29 mammograms from the MIAS database [9]. Once the linear structures were detected, a selection was labelled by an expert radiologist into anatomically distinct classes: ducts, edges, fibrous tissue, skin folds, spicules, vessels and others. Linear structures were randomly selected, taking the detected line strength into account, so that for each class the total number of profiles obtained approximately represented their prevalence in mammograms. In addition to providing the anatomical annotation, the radiologist also gave an indication for the width of the linear structures.

\subsection{Profile Width}

A comparison between the width as provided by the Line Operator and that as provided by the radiologist can be found in Fig. 1. Large deviations are possible and the distributions tend to be skewed to the negative side (indicating that the width provided by the radiologist tended to be larger than the width derived from the Line Operator).

Instead of using the raw scale from the Line Operator it is also possible to obtain a scale distribution for each linear structure and derive a more robust width. We have used the median width from the distribution for each structure. The reason we assume this to be more robust is indicated in Fig. 1, which also shows the deviation between the annotated widths and those derived from the median scale as provided by the Line Operator for linear structures. These results indicate that the distribution becomes closer to that given by the radiologists. 


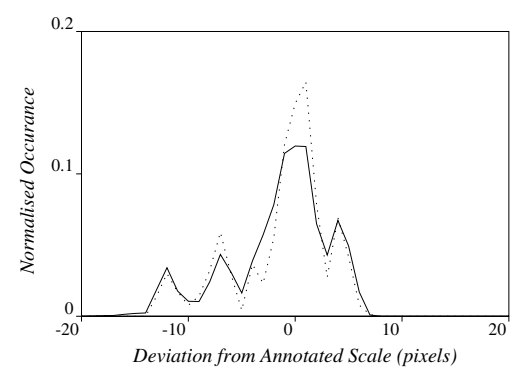

Fig. 1. Deviation between the annotated widths (range 2 to 30 pixels) and those derived from the scale as provided by the Line Operator for individual profiles (continuous lines) and those derived from the median scale as provided by the Line Operator for linear structures (dashed lines).

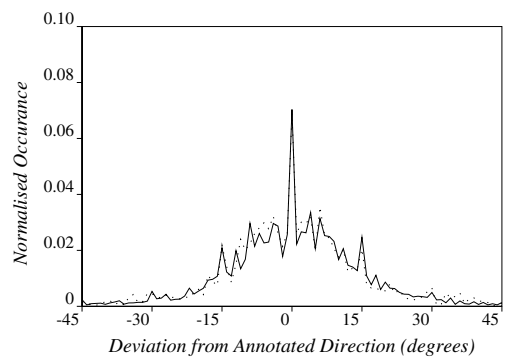

Fig. 2. Deviation between the annotated orientation and those derived from the orientation as provided by the Line Operator for individual profiles (continuous lines) and those derived from the median orientation as provided by the Line Operator for linear structures (dashed lines).

\subsection{Profile Orientation}

A comparison between the orientation as provided by the Line Operator and that as provided by the annotations can be found in Fig. 2. As for the width of the linear structures (see previous section) there is a wide distribution. These direct results from the Line Operator can be compared with the median orientation as obtained for the linear structures. Improvements in the orientation are not as clear as for the width.

\subsection{Extracting Profiles}

Cross-sectional profiles were obtained by taking the normal to the orientation found by the line operator and bilinear interpolation was used to sample greylevel values from the original mammograms. The cross-sectional length of the profiles was taken to be three times the width of the linear structure. To use these variable length profiles for statistical analysis, each profile was re-scaled to a constant number of pixels, 30 in this case, allowing each observation to be represented by an equal number of elements.

\subsection{Profile Preprocessing}

Instead of using the profiles directly it is also possible to use information derived from them. The motivation for such an approach is that a raw grey-level profile 
provides not only shape information but also describes the local background grey-level and contrast.

The grey-level values from the original mammogram were represented on a logarithmic scale (to represent tissue thickness). If a local background grey-level gradient existed this was removed by subtracting the gradient as obtained by a simple linear fit to the raw profile. In addition to remove the remaining effects of the grey-level background the mean grey-level was subtracted. To remove greylevel variations within the profiles normalisation with respect to the standard deviation of the grey-level was used. After this preprocessing the resulting profile should contain only the shape information.

\subsection{Profile versus Linear Structure Classification}

Using statistical modelling we obtain a class probability for every profile. However, we are in general interested in the classification of the linear structures. This can be obtained by a simple summation of the probabilities of the profiles (multiplication is more appropriate but resulted in probabilities equal to zero for the majority of structures).

\section{Results for Annotated Profiles}

Models were trained on a dataset comprising 318 linear structures (47,812 profiles). To avoid bias the modelling was applied using a three-way split of the dataset (on an image basis). In addition, to avoid bias between left and right mammograms all profiles were included twice for the modelling, once in the original format, but also in reverse order.

As we are interested in the detection of the linear structures that are associated with spiculated lesions we will restrict the classification results to the anatomical class of spicules. This means that for the classification results presented the spicules are regarded as the targets and will indicate the true positives and false negatives, while the other classes are regarded as the non-targets and will indicate the false positives and true negatives. Similar results could be presented for the other anatomical classes.

We have investigated two variations on the input information from the profiles that was used to obtain the classification results; classification based on the full profile and classification based on the PCA data derived from the profiles (using those principal components that covered $99 \%$ of the data variance). Two different artificial neural networks were used. The first network used 30 input nodes to represent the elements of the profiles. The second network used a lower number of input nodes, the exact value being determined by the PCA modelling. At the output level (i.e. the classification level) we used two nodes representing the class and non-class options. 

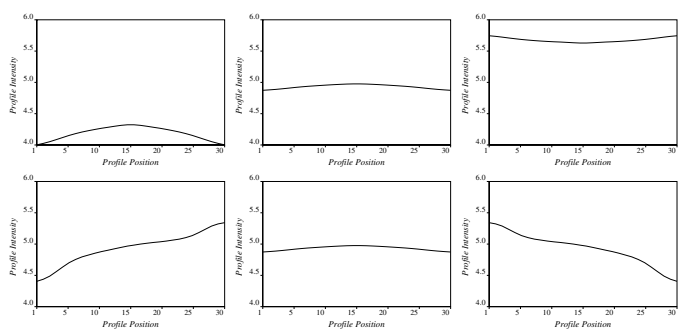

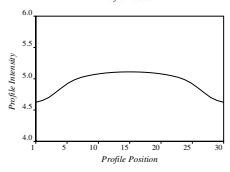

-2 sd

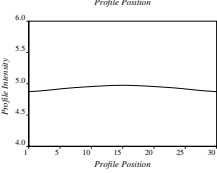

mean

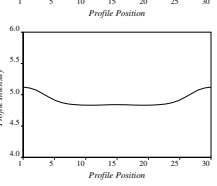

+2 sd

Fig. 3. Annotated Profiles: The mean (centre column) and first three (from top to bottom) principal components ( \pm 2 standard deviations) of the PCA model based on the raw profiles.

\subsection{Principal Component Analysis}

For models based on the raw profiles the cumulative variance associated with the first five principal components are respectively: 85.5\%, 95.3\%, 98.8\%, 99.4\% and 99.6\% . Fig. 3 shows the mean profile and the first three principal components of a PCA model based on raw profiles. The first principal component indicates a basic change in the mean grey-level of the profiles. The second principal component shows changes in the gradient of the background grey-level on which the profile is situated. The higher principal components capture changes in the shape of the profiles.

For models build using the normalised profiles the cumulative variance associated with the first five principal components are respectively $19.1 \%, 35.0 \%$, $49.4 \%, 58.2 \%$ and $65.0 \%$. The effect of the first three principal components and the mean profile are shown in Fig. 4. All the three principal components indicate variations in the shape of the profiles, without any of the effects of the background present. The first principal component seems to be an indication of the overall shift of the profile from left to right. The second and third principal components indicate changes in the width and multi-modal effects of the profiles.

\subsection{Classification}

ROC curves for linear and non-linear classification of the linear structures based on the original profiles are shown in Fig. 5. The classification shows a significant improvement for the non-linear classification of the linear structures.

ROC curves for linear and non-linear classification based on the normalised profiles are shown in Fig. 6. This indicates an improvement when the non-linear modelling is used. In saying this it has to be kept in mind that the improvements 

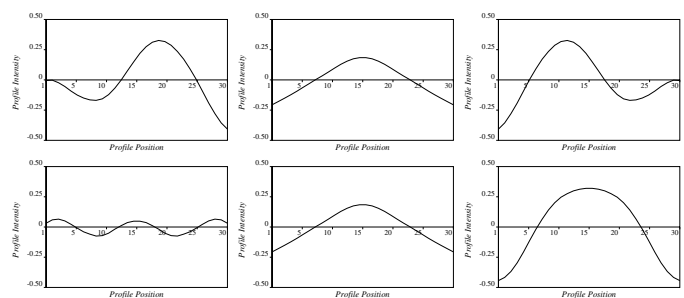

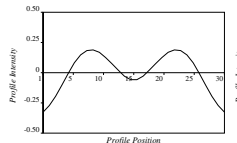

$-2 \mathrm{sd}$

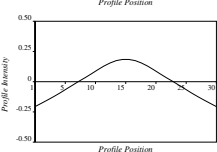

mean

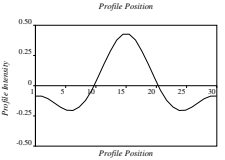

+2 sd

Fig. 4. Annotated Profiles: The mean (centre column) and first three (from top to bottom) principal components ( \pm 2 standard deviations) of the PCA model based on the normalised profiles.

are small and that this preprocessing of the profiles does not result in large discrimination effects.

These results indicate that the shape of the profile on its own might not be sufficient for the classification of anatomical linear structures. But when the raw profiles are used the results look very promising, with optimum ROC results of $80 \%$ True Positives at the cost of $35 \%$ False Positives. The differences between the classification based on the raw and normalised profiles are an implicit indication of the classification potential of the width, mean grey-level and standard deviation of the profiles.

\section{Results for Automaticly Extracted Profiles}

For an automatic system the width and orientation of the linear structures has to be determined by a computer vision approach. In this section we present results obtained using the scale and orientation provided by the Line Operator [8]. The median scale for each structure is used (see Section 2.2). The preprocessing of the profiles is identical to those described in Section 2.5.

\subsection{Principal Component Analysis}

For the PCA model based on the raw profiles the cumulative variance associated with the first five principal components are respectively: 95.0\%, 98.3\%, 98.9\%, $99.2 \%$ and $99.5 \%$. The mean profile and the principal components of a PCA model based on the raw profiles are very similar to those presented using the annotated width and orientation as presented in Fig. 3.

For models build using the normalised profiles the cumulative variance associated with the first five principal components are respectively $19.5 \%, 37.0 \%$, $48.9 \%, 57.1 \%$ and $63.4 \%$. Again, the resulting PCA models are very similar to 


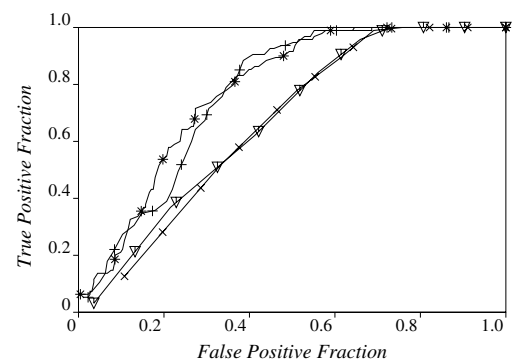

Fig. 5. Annotated Profiles: ROC linear structure classification based on the original profiles for spicule/non-spicule classification. Where $\nabla$ : linear classification based on the full profile, $\times$ : linear classification based on the PCA data, *: nonlinear classification based on the full profile, and +: non-linear classification based on the PCA data.

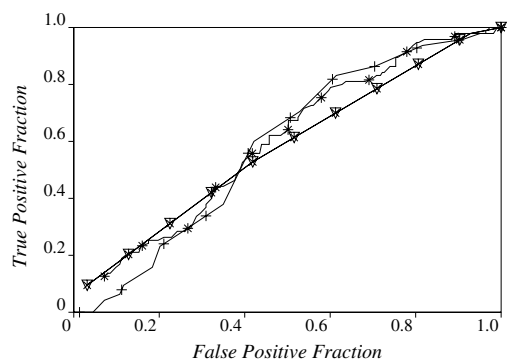

Fig. 6. Annotated Profiles: ROC linear structure classification based on preprocessed profiles for spicule/non-spicule classification. Where $\nabla$ : linear classification based on the full profile, $\times$ : linear classification based on the PCA data, *: nonlinear classification based on the full profile, and +: non-linear classification based on the PCA data.

those presented for the profiles extracted using the annotated width (see Fig. 4), except for the change-over of the first two principal components (both of them covering about $19 \%$ of the variation in the profile data).

\subsection{Classification}

ROC curves for linear and non-linear classification based on the original profiles is shown in Fig. 7. This shows a small improvement for the non-linear classification of the linear structures.

ROC curves for linear and non-linear classification based on the normalised profiles is shown in Fig. 8. Classification into class/non-class indicates no improvement when the non-linear method is used.

The comparison between the results presented in this section and those presented in Section 3.2 indicate large differences with an overall degradation of the classification results. The only difference between the two approaches has been a change in the width and orientation of the profiles that were extracted. It seems that the width as selected by the radiologists results in profiles which are easier to classify. 


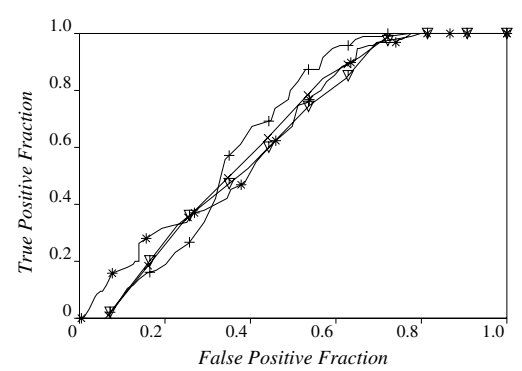

Fig. 7. Median Line Operator Profiles: ROC linear structure classification based on the original profiles for spicule/nonspicule classification. Where $\nabla$ : linear classification based on the full profile, $x$ : linear classification based on the PCA data, *: non-linear classification based on the full profile, and +: non-linear classification based on the PCA data.

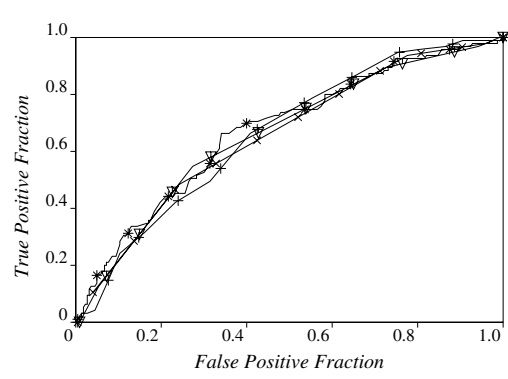

Fig. 8. Median Line Operator Profiles: ROC linear structure classification based on preprocessed profiles for spicule/nonspicule classification. Where $\nabla$ : linear classification based on the full profile, $\times$ : linear classification based on the PCA data, *: non-linear classification based on the full profile, and +: non-linear classification based on the PCA data.

\section{Discussion and Conclusions}

An automated technique for detecting specific anatomical types of linear structures in digital mammograms has been described. We have demonstrated how principal component analysis can be used to successfully model the shape of the cross-sectional intensity profiles of the anatomically different linear structures. In addition non-linear aspects of profile classification have been investigated.

A three-way split classification experiment demonstrated anatomical classification with a significantly better than random correct classification for the spicule profiles. A correct classification rate of $80 \%$ for spicules/non-spicules could be achieved (at a false positive percentage of $\sim 35 \%$ ), which will clearly be useful for the verification of potential spiculated lesions and architectural distortions. In similar experiments, good classification results have also been obtained for vessels and ducts. The potential of this technique for discriminating between ducts and vessels to reduce the number of false positives generated by automated micro-calcification detection algorithms is clear [7]. The detection and classification of ducts is likely to provide useful information for automated techniques attempting to locate the nipple.

However, it does not seem to be the shape of the profile that contributes most significantly to the classification of the linear structures. The width, mean grey-level and standard deviation of the profiles are implicated as being good parameters for classification of the profiles. As soon as these factors have been 
removed from the profiles the classification potential is reduced, but still better than random, indicating some useful classification potential for the shape of the profiles.

Large classification differences occurred when automaticly determined width and orientation were used when compared with the results obtained when these parameters were derived from the annotations provided by the radiologist. This indicates that improved classification results could be obtained if the width and orientation could be extracted more reliably.

In summary, the algorithms presented in this paper provide an automatic technique for locating and successfully classifying anatomically important linear structures of which spicules were used as an example. Such classification can be employed to verify automatically detected potential abnormalities such as microcalcification clusters, spiculated lesions and architectural distortions. In addition, the technique can be used to provide strength images relating to specific classes of abnormality; spicule strength images can for example be used to improve the accuracy of spiculated lesion detection algorithms [1,4,5]. The technique can also be used to reduce the required computation time by examining only those pixels that are classified into a given class.

\section{References}

1. R. Zwiggelaar, T.C. Parr, J.E. Schumm, I.W. Hutt, S.M. Astley, C.J. Taylor, and C.R.M. Boggis. Model-based detection of spiculated lesions in mammograms. Medical Image Analysis, 3(1):39-62, 1999. 263, 264, 271

2. S.M. Astley, R. Zwiggelaar, C. Wolstenholme, and C.J. Taylor. Prompting in mammography: how accurate must prompt generators be? $4^{\text {th }}$ International Workshop on Digital Mammography, Nijmegen, The Netherlands:347-354, 1998. 263

3. H.-P. Chan, K. Doi, C.J. Vyborny, R.A. Schmidt, C.E. Metz, K.L. Lam, T. Ogura, $\mathrm{Y}$. $\mathrm{Wu}$, and $\mathrm{H}$. MacMahon. Improvement in radiologists' detection of clustered microcalcifications on mammograms. Investigative Radiology, 25(10):1102-1110, 1990. 263

4. W.P. Kegelmeyer, J. Pruneda, P. Bourland, A. Hills, M. Riggs, and M. Nipper. Computer-aided mammographic screening for spiculated lesions. Radiology, 191:331-337, 1994. 263, 264, 271

5. N. Karssemeijer and G.M. te Brake. Detection of stellate distortions in mammograms. IEEE Transactions on Medical Imaging, 15(5):611-619, 1996. 263, 264, 271

6. L. Tabar and P.B. Dean. The Mammographic Teaching Atlas. Georg Thieme Verlag, Stuttgart, 1985. 263

7. T. Ema, K. Doi, R.M. Nishikawa, Y. Jiang, and J. Papaioannou. Image feature analysis and computer-aided diagnosis in mammography: reduction of false-positive clustered microcalcifications using local edge-gradient analysis. Medical Physics, 22(2):161-169, 1995. 263, 270

8. R. Zwiggelaar, T.C. Parr, and C.J. Taylor. Finding orientated line patterns in digital mammographic images. In Proceedings of the $7^{\text {th }}$ British Machine Vision Conference, pages 715-724, Edinburgh, UK, 1996. 264, 268 
9. J. Suckling, J. Parker, D. Dance, S. Astley, I. Hutt, C. Boggis, I. Ricketts, E. Stamatakis, N. Cerneaz, S. Kok, P. Taylor, D. Betal, and J. Savage. The mammographic images analysis society digital mammogram database. In Dance Gale, Astley and Cairns, editors, Digital Mammography, pages 375-378. Elsevier, 1994. 264 